\title{
Hemostatic completion of percutaneous nephrolithotomy using electrocauterization and a clear amplatz renal sheath
}

\author{
Ho Song Yu ${ }^{1}$, Ji Won Ryu ${ }^{1}$, Sun-Ouck Kim ${ }^{1}$, Taek Won Kang ${ }^{1}$, Dong Deuk Kwon ${ }^{1}$, Kwangsung Park ${ }^{1}$, \\ Kyung Jin $\mathrm{Oh}^{1}$
}

${ }^{1}$ Department of Urology, Chonnam National University Medical School, Gwangju, Republic of Korea

\begin{abstract}
Background and Purpose: A tubeless PCNL can reduce postoperative pain, the need for analgesics, hospital stay, and postoperative urinary leakage. However, perioperative or delayed bleeding remains the primary postoperative concern. We demonstrate a simple and cost-effective method to develop a clear nephrostomy tract after completion of a tubeless PCNL. Materials and Methods: Four consecutive patients with renal calculi $>3 \mathrm{~cm}$ underwent a tubeless PCNL. We used a 24 Fr nephroscope and a $24 \mathrm{Fr}$ transurethral resectoscope. Intraoperative urologist-directed percutaneous renal access was performed under fluoroscopy. After calculi removal, active bleeders were identified via a clear Amplatz renal sheath. The sheath provided excellent visualization of the nephrostomy tract for the detection of bleeders and surrounding structures. Bleeders were electrocauterized using a roller barrel electrode. During extraction of the renal sheath, the surgeon can confirm hemostasis in the tract and apply intermittent suction.

Results: Bleeding primarily originated from the torn calyeceal mucosa and the parenchyma. Tract electrocauterization was successful. All patients had mild hematuria, which resolved within two days. The average hemoglobin decrease was $1.65 \mathrm{~g} / \mathrm{dL}(0.8-2.1)$ and no patients required a transfusion. No perioperative complications occurred. On postoperative day 2, the patients could ambulate without a Foley catheter. During three months of follow-up, delayed bleeding or percutaneous urine leakage did not occur.

Conclusions: Electrocauterization with a roller barrel electrode and a clear Amplatz renal sheath is an effective method to obtain hemostasis after completion of a PCNL. Our technique is cost-effective and readily adapted without the need for additional instruments.
\end{abstract}

\section{ARTICLE INFO}

Available at: http://www.intbrazjurol.com.br/video-section/video-library/yu_170_171/

Int Braz J Urol. 2016; 42 (Video \#2): 170-1

Submitted for publication:

August 07, 2015

Accepted after revision:

October 18, 2015
Correspondence address:

Kyung Jin Oh, MD

Department of Urology Chonnam National University Medical

School

8 Hak-Dong, Dong-Gu

Gwangju, 501-757, Republic of Korea

Fax: + 82622 271-643

E-mail: exeokj@hanmail.net 


\section{EDITORIAL COMIMENT}

In the present video submission by $\mathrm{Yu}$ et al., the authors nicely depict a novel way bleeding encountered at the time of a percutaneous nephrolithotomy can be controlled using electrocautery and a clear Amplatz sheath. This is clearly one of the most challenging clinical scenarios encoun- tered at time of such procedures can be addressed minimizing perioperative morbidity and the necessity for possible additional ancillary procedures. Developing innovative solutions to manage such surgical complications are strongly encouraged and I congratulate the authors in doing so particularly as this requires readily available surgical equipment at our disposition.

Philippe E. Spiess, MD Associate Member, Department of Genitourinary Oncology Moffitt Cancer Center Tampa, FL, USA Video Section Editor, International Brazilian Journal of Urology E-mail: philippe.spiess@moffitt.org 